\title{
Development of an Axial Flux Type PM Synchronous Motor With the Liquid Nitrogen Cooled HTS Armature Windings
}

\author{
Hidehiko Sugimoto, Teppei Tsuda, Takaya Morishita, Yoshinori Hondou, Toshio Takeda, Hiroyuki Togawa, \\ Tomoya Oota, Kazuya Ohmatsu, and Shigeru Yoshida
}

\begin{abstract}
We developed an axial gap permanent magnet type high temperature superconducting synchronous motor (PMHTSSM) at liquid nitrogen temperature. There are two distinctive differences between this motor and the conventional HTS motors. The permanent magnet is applied to the field, iron cores and HTS wires are used as the armature. The motor has eight field poles, and six armature windings. The armature is put between the two fields. The field composes eight permanent magnets which are arranged as a circle. Four poles share three armature coils and there are two coils for every phase. The BSCCO wires, which are applied to armature windings, work at the temperatures of between $66 \mathrm{~K}$ and $70 \mathrm{~K}$. The rated output is $400 \mathrm{~kW}$ at $250 \mathrm{rpm}$. It is known that superconducting wires have $\mathrm{AC}$ loss if they are operated at alternating magnetic fields. That is why there are few motors developed with superconducting armature windings. Also, there is no report of motors applying HTS wires such as BSCCO wires to armature windings. Furthermore, this motor adopted the revolving field type structure, so the brushes were not needed and the cooling system can be fixed. Also because of the axial-flux type, the structure of the cryostat can be simplified.
\end{abstract}

Index Terms-Axial flux type, BSCCO HTS wire, permanent magnet, permanent magnet HTS synchronous motor.

\section{INTRODUCTION}

$\mathbf{W}$ E developed a liquid nitrogen cooled axial flux type Permanent Magnet High Temperature Superconducting Synchronous Motor (PMHTSSM). In order to pursuit a high efficiency and small size HTSM and PM synchronous machine, this motor used liquid nitrogen temperature HTS wires as the armature windings instead of copper wires.

Since the permanent magnets are used in the field, the armature iron core is necessary to obtain large magnetic flux density.

Manuscript received August 28, 2006.

H. Sugimoto, T. Tsuda, T. Morishita, and Y. Hondou are with the Department of Electrical and Electronic Engineering, University of Fukui, 901 8507, Japan (e-mail: sugimoto@fuee.fukui-u.ac.jp).

T. Takeda, H. Togawa, and T. Oota are with Superconductive Ship Propulsion System Dev., Ishikawajima-Harima Heavy Industries Co., Ltd. 100-8182 Japan (e-mail: toshio_takeda@ihi.co.jp).

K. Ohmatsu is with HTS R\&D Department, Electric Power \& Energy Research Laboratories, Sumitomo Electrical Industries, Ltd. Osaka, 554-0024, Japan (e-mail: ohmatsu@ sei.co.jp).

S. Yoshida is with the Engineering Department, Cryogenic Project, Development \& Engineering, Taiyo Nippon Sanso Corporation. Ibaraki, 300-2611, Japan (e-mail: Sgr.Yoshida@tn-sanso.co.jp).

Color versions of one or more of the figures in this paper are available online at http://ieeexplore.ieee.org.

Digital Object Identifier 10.1109/TASC.2007.899959
There are two advantages to apply the iron core to armature HTS windings. First, it reduced the flux to penetrate HTS wire, which leads to reduced AC loss. Secondly, it helped to reduce force acting on HTS wires. In recent years, there are great advances on the Neodymium magnet. It is applied to the field since it can produce large flux density. The revolving field type structure can employ relatively simple and efficient refrigeration systems, also the iron core does not need to be cooled, these methods help to reduce iron losses. By the facts mentioned above, a small size HTSSM has been built. The efficiency of the motor is under testing now.

The performance of HTS wires at AC conditions is better than that of Low Temperature Superconducting (LTS) wires. Generally, the liquid helium or Neon is used to cool LTS machines. HTS machines have advantages in operating temperature and lower loss. Also, advances in the development of HTS have resulted in high critical current. Currently, HTS rotation machines are developing worldwide.

There are several superconducting PM motors and fully superconducting motors have been reported in [1]-[3]. They all used LTS wires at helium temperature as their armatures. While, all the basic structures of the HTS rotating machines that have been reported used the HTS wires for the field windings and used the copper wires for the armature windings. Most of the HTS rotating machines with these structures employed the neon or helium as the coolant.

Moreover, there are several examples of HTS machines operating at the liquid nitrogen temperature level reported, such as the machines in [4], [5]. The machine in [4] is a radial flux type generator where the BSCCO wires are applied to the magnetic field windings. Also, iron cores were applied to the field to concentrate the flux. As a result, the critical current of HTS wires increased. The HTS windings and iron cores were cooled at the same time, which leads to cooling loss and iron loss increased. Due to the influence of the armature space harmonics, the iron core in DC conditions produces iron loss. The cooled iron cores leads to the higher eddy current loss due to increased eddy current. Besides, the cooling loss of iron core is increased too. Furthermore, as it applies the revolving field type structure, the cooling system has to rotate, so the coolant flows between the fixed part and stationary part. Consequently, it increases the complexity of the cooling system such as the cryostat part.

These kinds of problems strongly influence the miniaturization of the HTSM, which is the main objective of the superconducting applications. 
TABLE I

BASIC SPECIFICATIONS FOR THE PMHTSSM

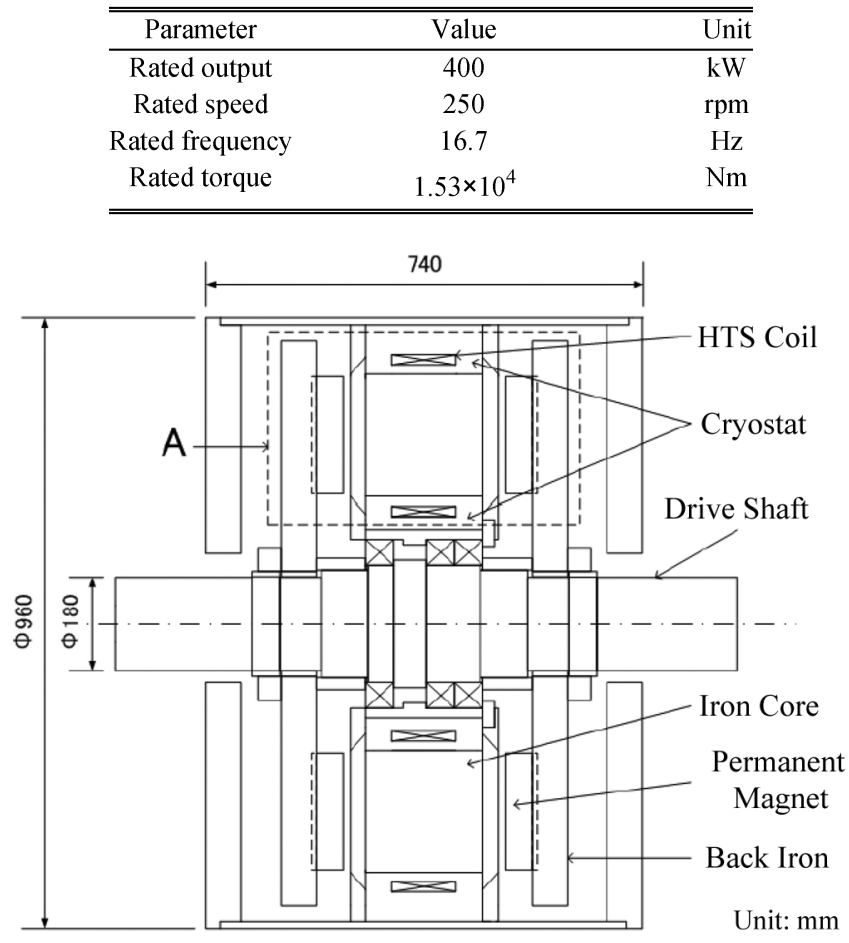

Fig. 1. Sketch of the $400 \mathrm{~kW}$ PMHTSSM.

In order to solve these problems, we carried out the research of a new type of HTSM which uses the liquid nitrogen as the coolant.

This paper presents the structures, design of the HTSM, as well as the analytical results by finite elements method (FEM) and the test results of the trial motor.

\section{IDEAS OF DESIGN AND ELECTROMAGNETIC FIELD ANALYSIS}

\section{A. Ideas of Design}

The Table I shows the basic specifications for the PMHTSSM. The Fig. 1 shows the schematic drawing of PMHTSSM. The permanent magnets are used in the field, BSCCO wires are used in the armature windings. The cryostat surrounds iron cores which are fixed by arranging Fiber reinforced plastic (FRP) at two sides. The armature coils consist of eighteen double pancakes, which are stacked together on a central structural form. The coils are connected in parallel one by one.

The Fig. 2 represents the relationships between the current and the flux density at difference temperatures, where curve (a) stands for the case of horizontal direction, while the curve (b) stands for the case of vertical direction. Both relationships are set up by measured values offered by the producer of the HTS wires. $\mathrm{LN}_{2}$ is cooled down to $66 \mathrm{~K} \sim 70 \mathrm{~K}$ to increase the critical current of the HTS wires. The features of the HTSM that we designed are shown as follows:

1) Compared to iron cores, PM has higher reluctance for fluxes produced by the armature, thus the armature reaction is reduced. Also, the flux density on the HTS wires is reduced too.

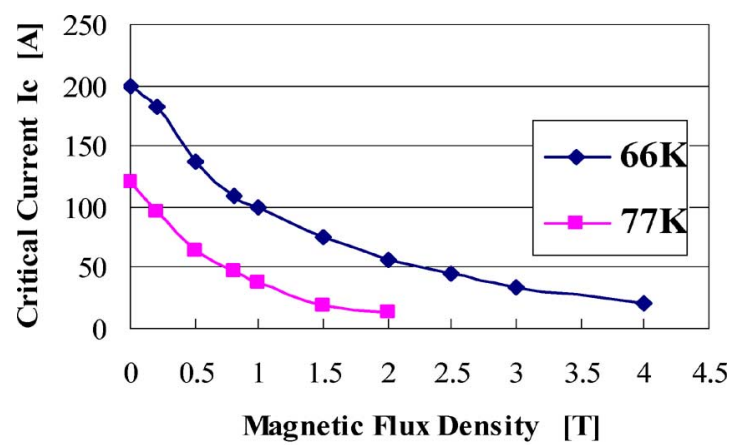

(a)

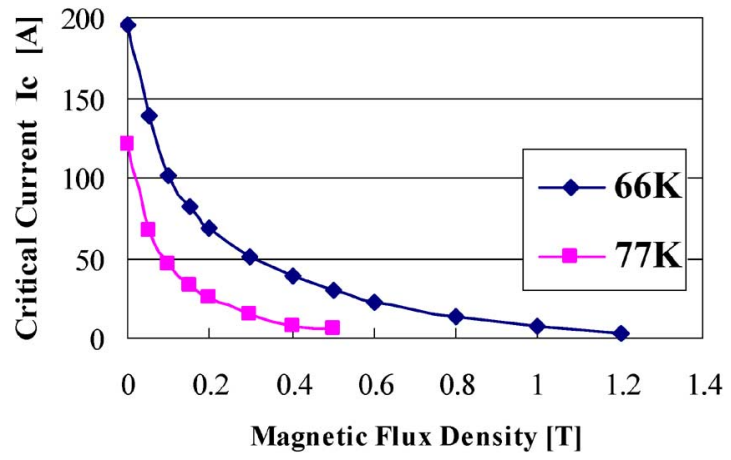

(b)

Fig. 2. Relationships between the current and magnetic flux density at difference temperatures of the BSCCO HTS wire: (a) Horizontal direction, (b) vertical direction.

2) The eddy currents in the cryostat are eliminated by using FRP as the cryostat material.

3) As iron cores are arranged at the center of the armature windings, the alternating magnetic field which acts on HTS wires are weakened. This helps to reduce the AC loss of the HTS windings. Also, the force that acts on the HTS wires can be reduced, and the critical current can be increased. Meanwhile, the eddy current of the iron core is reduced because it is a stacked core.

4) We adopted the axial flux type to simplify the cryostat.

5) Iron cores can be operated at room temperature due to the revolving field is adopted. So, the iron losses as well as the cooling losses of iron cores can be reduced. Moreover, the brushes do not need, back yoke's iron loss can be reduced and the cooling system can be simplified. A simpler cooling system leads to a smaller HTS machine.

6) This HTSM is planned to be used for the ship propulsion. The propeller has to be operated at low speed because it does not employ reduction gear to decrease its rotational speed. The frequency is $16.7 \mathrm{~Hz}$ at the rated speed, so the hysteresis losses, eddy current losses and the AC losses can be reduced because of this low frequency.

\section{B. Analysis}

We carried out the magnetic field analysis of the locked rotor test by FEM on the basis of designed model. The results are shown as follow. 


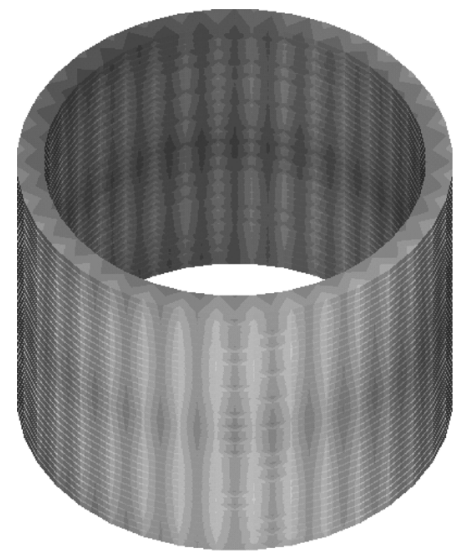

(a)
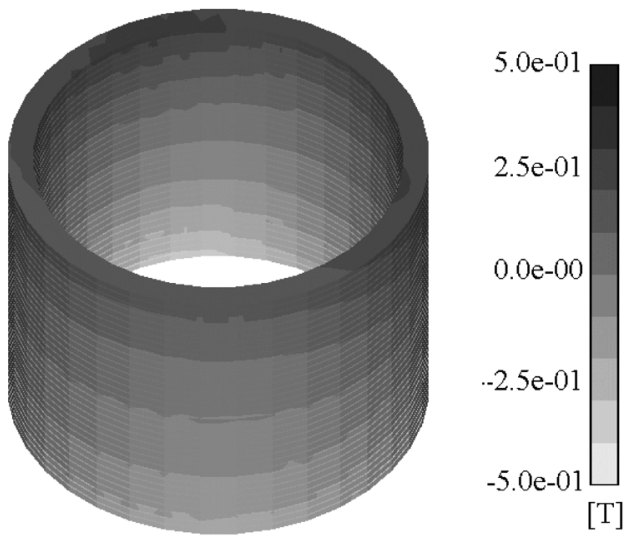

(b)

Fig. 3. Contour figure of the flux density penetrating HTS wires: (a) Horizontal direction, (b) vertical direction.
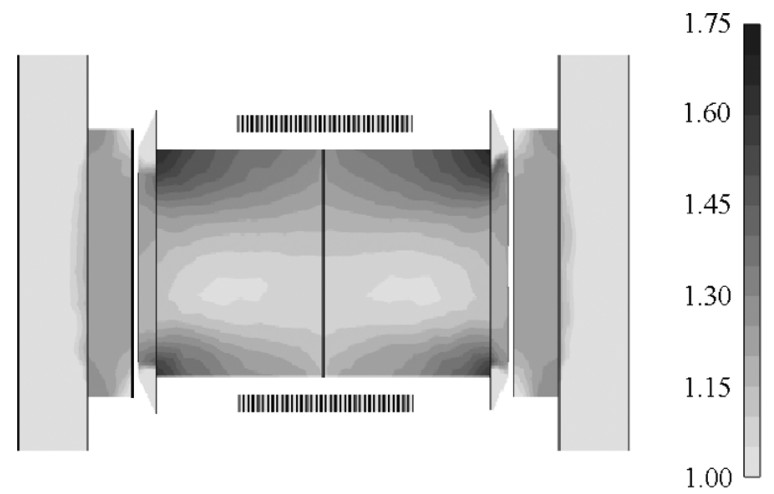

[T]

Fig. 4. Contour figure of the field flux density in the shalf's axial direction.

In this analysis, the motor torque is $1.6 \mathrm{kNm}$ while the rated armature current is $22.6 \mathrm{~A} /$ per HTS wire, which equals to 419 $\mathrm{kW}$ at $250 \mathrm{rpm}$. The diameter of the motor is $960 \mathrm{~mm}$, and width is $740 \mathrm{~mm}$. All these values meet the requirements of the design.

Fig. 3 and Fig. 4 show the analytical results, where Fig. 3(a) represents the flux density on the HTS wires at the horizontal direction and Fig. 3(b) represents the flux density on HTS wires at the vertical direction. According to the analytical result, the maximum flux density is $0.09 \mathrm{~T}$ at horizontal direction, and is $0.45 \mathrm{~T}$ at vertical direction. On the other hand, according to Fig.

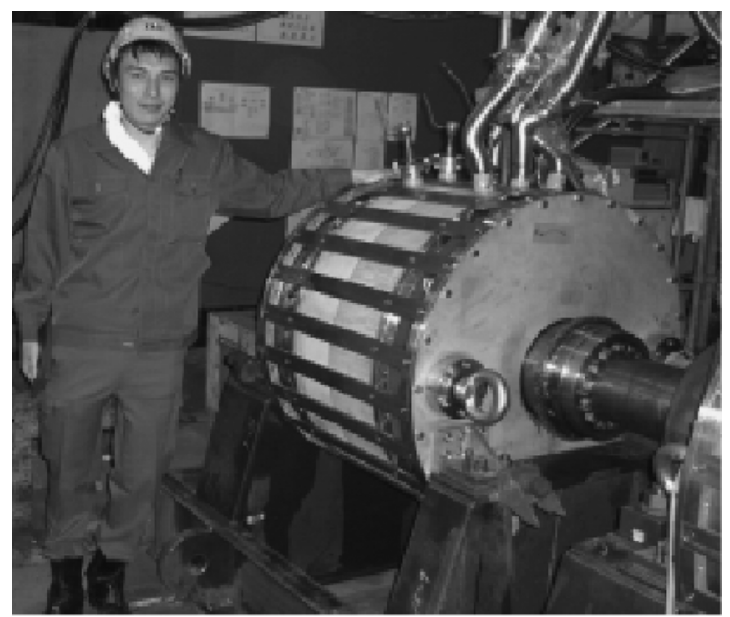

Fig. 5. Photograph of trial PMHTSSM.

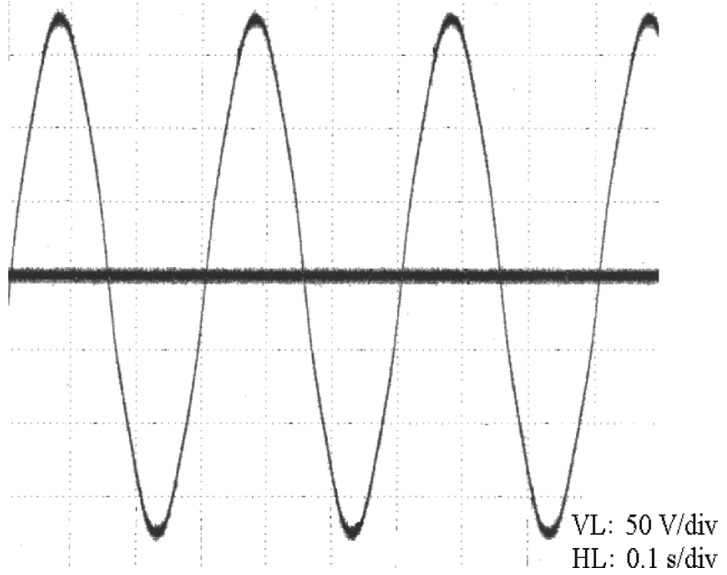

Fig. 6. Induced electromotive force's waveform at $50 \mathrm{rpm}$.

2 , the critical flux density at horizontal direction is $3.1 \mathrm{~T}$, and is $0.47 \mathrm{~T}$ at vertical direction. It is clearly that the flux densities do not exceed the critical flux density.

Fig. 4 is the contour figure of the field flux density of the iron core. The iron core and the surrounding armature winding are shown as part A in Fig. 1. It can be found that the average magnetic flux density of the surrounded iron core at the axial direction is $1.2 \mathrm{~T}$. This value is large enough to meet the design of the HTSM.

\section{RESUlts OF MOTOR TESTING}

In this chapter, we are going to compare the test results and the magnetic field analytical results, which are carried out under the same conditions. Fig. 5 shows the trial motor used in the tests, and the results are shown as follows:

\section{A. Induced Electromotive Force Test}

Fig. 6 shows the waveform of induced electromotive force at $50 \mathrm{rpm}$ between W-U lines, which is sine wave. The induced electromotive force is $618 \mathrm{~V}$ in the analysis at the rated speed, while it is $623 \mathrm{~V}$ that are calculated on the base of test values, these two results are about the same. 


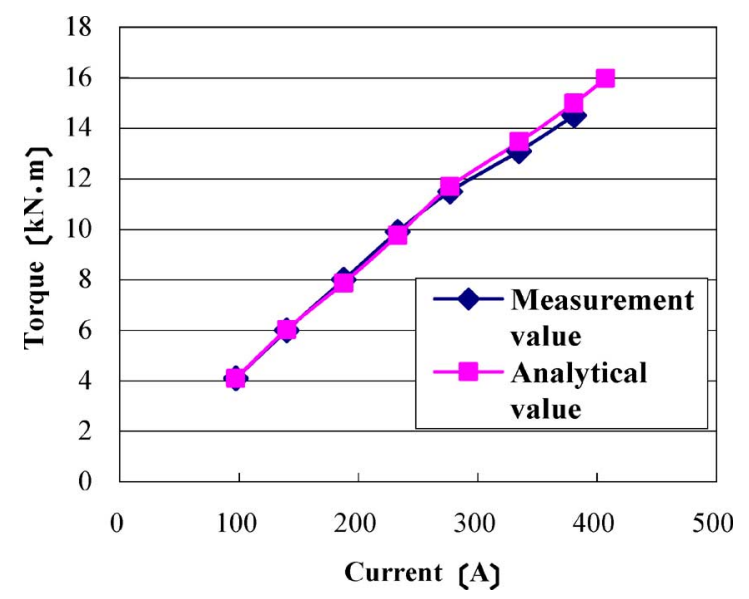

Fig. 7. Characteristics of the current and the torque.

\section{B. Locked Rotor Test}

Fig. 7 shows the relation between the current and the torque at the locked rotor test. It keeps linearity although there is a little bit of change due to the magnetic saturation. The test is done when the current values are less than 540 A since there is limit on the strength of the load equipments. At this time, the torque is 14.5 $\mathrm{kNm}$, equivalent to the $380 \mathrm{~kW}$ output at $250 \mathrm{rpm}$. Referring to the results that were obtained from the locked rotor test and the analysis, the object of $400 \mathrm{~kW}$ was reached with no doubts.

\section{Acceleration and Deceleration Test}

Finally, Fig. 8 shows the results of the acceleration and deceleration tests. From this figure, we can find that the command value is similar to the detection value. It means that the acceleration and deceleration are smooth.

\section{CONCLUSION}

According to the test results, it shows that the tested motor has the similar performance with that in the analysis. In this HTSM, the cooling system is simplified and brushes are not needed because of the revolving field type and axial flux type structure. Besides, as far as the AC losses caused by the utilizing of HTS wires in the armature are concerned, we adopted the methods

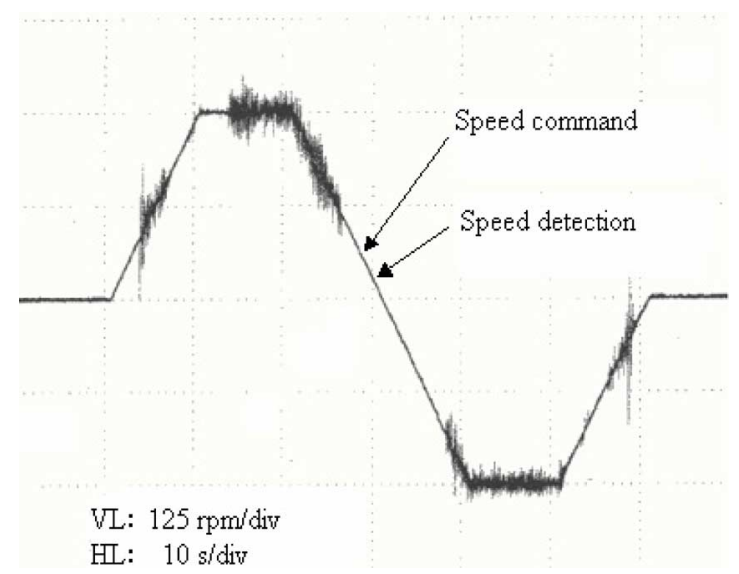

Fig. 8. Characteristic of the acceleration and deceleration for fourth quadrant drive distribution.

such as reducing the flux penetrates windings by arranging the iron core or increasing the reluctance to the armature by employing permanent magnets.

This HTS motor proves the feasibility of the method adopted to achieve the high efficiency and miniaturization of the new type HTSM in which the permanent magnets are used in the fields and HTS windings are used in the armature.

The load experiments and experiments of testing AC loss will be carried out. Also, the manufacture and verification of the machine for practical use are under planning.

\section{REFERENCES}

[1] O. Tsukamoto, N. Amemiya, and K. Yamagishi, "Development and electrical test of a $30 \mathrm{kVA}$ class fully superconducting generator," IEEE Trans. Magn., vol. 30, no. 4, pp. 2308-2311, Jul. 1994.

[2] P. Tixador and H. Daffix, "Conceptual design of an electrical machine with both low and high Tc superconductors," IEEE Trans. Appl. Supercond., vol. 7, no. 4, pp. 3858-3865, Dec. 1997.

[3] P. Tixador, F. Simon, H. Daffix, and M. Deleglise, "150-kW experimental superconducting permanent-magnet motor," IEEE Trans. Appl. Supercond., vol. 9, no. 2, pp. 1205-1208, Jun. 1999.

[4] M. K. Al-Mosawi, C. Beduz, and Y. Yang, "Construction of a 100 kVA high temperature superconducting synchronous generator," IEEE Trans. Appl. Supercond., vol. 15, no. 2, pp. 2182-2185, Jun. 2005.

[5] H. Sugimoto, T. Nishikawa, T. Tsuda, Y. Hondou, Y. Akita, T. Takeda, T. Okazaki, S. Ohashi, and Y. Yoshida, "Trial manufacture of liquid nitrogen cooling high temperature superconductivity motor," $2006 \mathrm{~J}$. Phys.: Conf. Series, vol. 43, pp. 780-783. 